\title{
Evaluation of Left and Right Ventricular Systolic and Diastolic Electromechanical Synchrony in Older People: a Population-Based Observational Study
}

\author{
R. LASZLO ${ }^{1}$, H. KONZ ${ }^{1}$, K. KUNZ ${ }^{1}$, D. DALLMEIER ${ }^{2}$, J. KLENK ${ }^{3}$, M. DENKINGER ${ }^{2}$, \\ W. KOENIG ${ }^{4}$, D. ROTHENBACHER ${ }^{3}$, J. M. STEINACKER ${ }^{1}$; FOR THE ACTIFE STUDY \\ GROUP
}

${ }^{1}$ Division of Sports and Rehabilitation Medicine, Ulm University, Ulm, Germany, ${ }^{2}$ AGAPLESION Bethesda Clinic, Geriatric Center Ulm/Alb-Donau, Ulm University, Ulm, Germany, ${ }^{3}$ Institute of Epidemiology and Medical Biometry, Ulm University, Ulm, Germany, ${ }^{4}$ Department of Cardiology, German Heart Center, Technical University Munich and DZHK (German Centre for Cardiovascular Research), Partner Site Munich Heart Alliance, Munich, Germany

Received July 28, 2016

Accepted June 2, 2017

On-line September 22, 2017

\section{Summary}

It is unknown whether physiological ageing also goes along with electromechanical asynchrony of contraction. Aim of the study was to evaluate synchrony of contraction in older people with ("non-healthy") or without ("healthy") evidence for structural cardiac disease. In 547 persons (age 76.7 \pm 5.5 years, 306 male, 241 female) recruited from a population-based cohort of the ActiFE-Ulm study including a random sample of people $\geq 65$ years old living in the region of Ulm, Germany, various PW- and TDI-Doppler based markers for asynchrony were obtained by echocardiography. Within a subgroup of 84 healthy subjects, at most minimal systolic and diastolic asynchrony was found. Concerning systolic asynchrony, similar observations were made within the non-healthy subgroup. However, extent of diastolic left ventricular intraventricular asynchrony and also - by tendency diastolic interventricular asynchrony was increased in comparison to the healthy subgroup. To conclude, no evidence that physiological ageing might go along with relevant left or right ventricular systolic or diastolic electromechanical asynchrony was found in our study. Furthermore, our population-based data support the results from other clinical studies with rather selected cohorts that structural heart diseases might go along with increased diastolic asynchrony.

\section{Key words}

Echocardiography • Asynchrony • Dyssynchrony • EC-coupling • Ageing $\bullet$ Population-based $\bullet$ Elderly $\bullet$ Older

\section{Corresponding author}

R. Laszlo, Division of Sports and Rehabilitation Medicine, Ulm University, Leimgrubenweg 14, 89070 Ulm, Germany. Fax: +49 731500 45303. E-mail: roman.laszlo@uniklinik-ulm.de

\section{Introduction}

Cardiac morphology and function is greatly affected by aging (Chiao et al. 2015, Karavidas et al. 2010). On cellular level, the total number of cardiomyocytes decreases accompanied by simultaneous hyperplasia of the remaining myocytes and the deposition of collagen between the cells. Cardiomyocytes present a reduction both in contraction and relaxation capability as a consequence of for example alterations in calcium homeostasis. In addition, altered calcium handling may also lead to age-related changes in excitation-contraction coupling (Feridooni et al. 2015). The cardiac conduction system is also subjected to ageing processes resulting in a higher incidence of both brady- and tachyarrhythmias (Chow et al. 2012, Mirza et al. 2012).

Intra- and/or interventricular electromechanical 
asynchrony of contraction can be a concomitant phenomenon of reduced cardiac pumping function (Carerj et al. 2009, Chan et al. 2008, Lafitte et al. 2006, Perez de Isla et al. 2008). This has also been shown in studies on cardiac resynchronization therapy (CRT) of patients with severe congestive heart failure in which echocardiographic parameters including cut-off values for evaluation of cardiac synchrony of contraction have been defined (Linde et al. 2012, Schuster et al. 2005).

We hypothesized that ageing-related alterations of excitation-contraction coupling may also result in intra- and/or interventricular electromechanical systolic or diastolic asynchrony of contraction. Therefore, aim of our study was to echocardiographically evaluate synchrony of contraction in older subjects who were recruited from a large population-based cohort.

\section{Methods}

The ActiFE study (Activity and Function in the Elderly with a focus on physical activity and co-morbidities) includes a random sample of initially 1506 people older than 65 years living in the region of Ulm, Germany, who were recruited between March 2009 and April 2010. Details have been described previously (Denkinger et al. 2010). Ethical approval was granted by the Ethical Committee of the University of Ulm. All participants gave written informed consent. The work described in the following has been carried out in accordance with The Code of Ethics of the World Medical Association (Declaration of Helsinki).

During the 3-years follow-up with a total participation of $834(55.4 \%)$ subjects, cardiovascular assessment including echocardiography was performed in a total of 688 (401 male, 287 female) persons (Fig. 1). The remaining subjects $(17.5 \%)$ refused participation. Cardiovascular diseases and risk factors were evaluated using a standardized questionnaire. A digital 12-lead resting ECG was registered during follow-up presentation. Height and body mass were surveyed using standard equipment. Physical activity (PA, steps/day among other parameters) was assessed by using an accelerometer (activPAL, PAL Technologies Ltd., Glasgow, UK), details have been described elsewhere (Denkinger et al. 2010).

Participants lay supine for at least five minutes before the echocardiographic examination. Thereafter, heart rate (HR) and blood pressure (BP) at rest were obtained. During echocardiography, the subject was lying in a left lateral decubitus position. By using a commercially available ultrasound system (Philips CX-50 with a S5-1 cardiac probe), all subjects were examined using a standard protocol following international guidelines (Lang et al. 2015). Left ventricular mass (Devereux) and left atrial volume (LAV) were calculated according to current recommendations (Lang et al. 2015) and were indexed to BSA (calculated using Mosteller's formula). Synchrony of contraction was evaluated by analyzing: 1) PW-Doppler curves within left and right ventricular outflow tract (method 1) and 2) TDI curves from three LV/RV areas (method 2, measurements were taken medial and lateral, one centimeter below mitral valve annulus and at the lateral free RV wall, one centimeter below tricuspid valve annulus, see also inset image of Figure 2B) (Faber et al. 2003, Linde et al. 2012, Perez de Isla et al. 2005, Perez de Isla et al. 2008, Quan et al. 2012, Rouleau et al. 2001, $\mathrm{Yu}$ et al. 2003, Yu et al. 2007). PW-Doppler- and TDI-curves were registered with a simultaneous superimposed ECG at a sweep speed of $100 \mathrm{~mm} / \mathrm{s}$.

For the evaluation of synchrony, both (method 1) or all three curves (method 2) were needed. However, not every single curve was obtainable in every subject due to sometimes impaired image quality. Subjects were only chosen for further analysis if evaluation of synchrony was possible by at least one of the two methods (meaning either a complete Doppler- or TDI-data set was obtainable). Therefore, 141 persons (95 male, 46 female) had to be excluded from further analyses (Fig. 1). Subjects with known atrial fibrillation (AF), coronary artery disease (CAD), or positive anamnesis for device implantation (pacemaker or ICD), wall motion abnormalities, reduced systolic function or diastolic function, NT-proBNP $>125 \mathrm{pg} / \mathrm{ml}$ (McMurray et al. 2012) as well as subjects with specific impairment of the cardiac conduction system in terms of complete or incomplete left or right bundle branch block were pooled in a subgroup of persons with evidence for structural heart disease, hereafter referred to as "non-healthy". Presence of AF was evaluated by resting electrocardiogram (ECG) or anamnesis. CAD was defined as positive response to "previous myocardial infarction", "coronary heart disease", CABG or stent implantation in a questionnaire. Reduced systolic function was assumed if LV-EF was $<52 \%$ (male) or $<54 \%$ (female) (Lang et al. 2015). Presence of diastolic function was defined as E/E'>15 (Nagueh et al. 2009). NT-proBNP was measured by Electrochemiluminescence 
(Elecsys 2010). Bundle branch blocks were defined according to recent recommendations (Surawicz et al. 2009). From the 547 persons (306 male, 241 female) analyzed in our study, 463 (264 male, 199 female) were considered not healthy according to our chosen definition. The remaining 84 persons (42 male, 42 female) were pooled in the subgroup "no evidence for structural heart disease", hereafter referred to as "healthy" (Fig. 1).

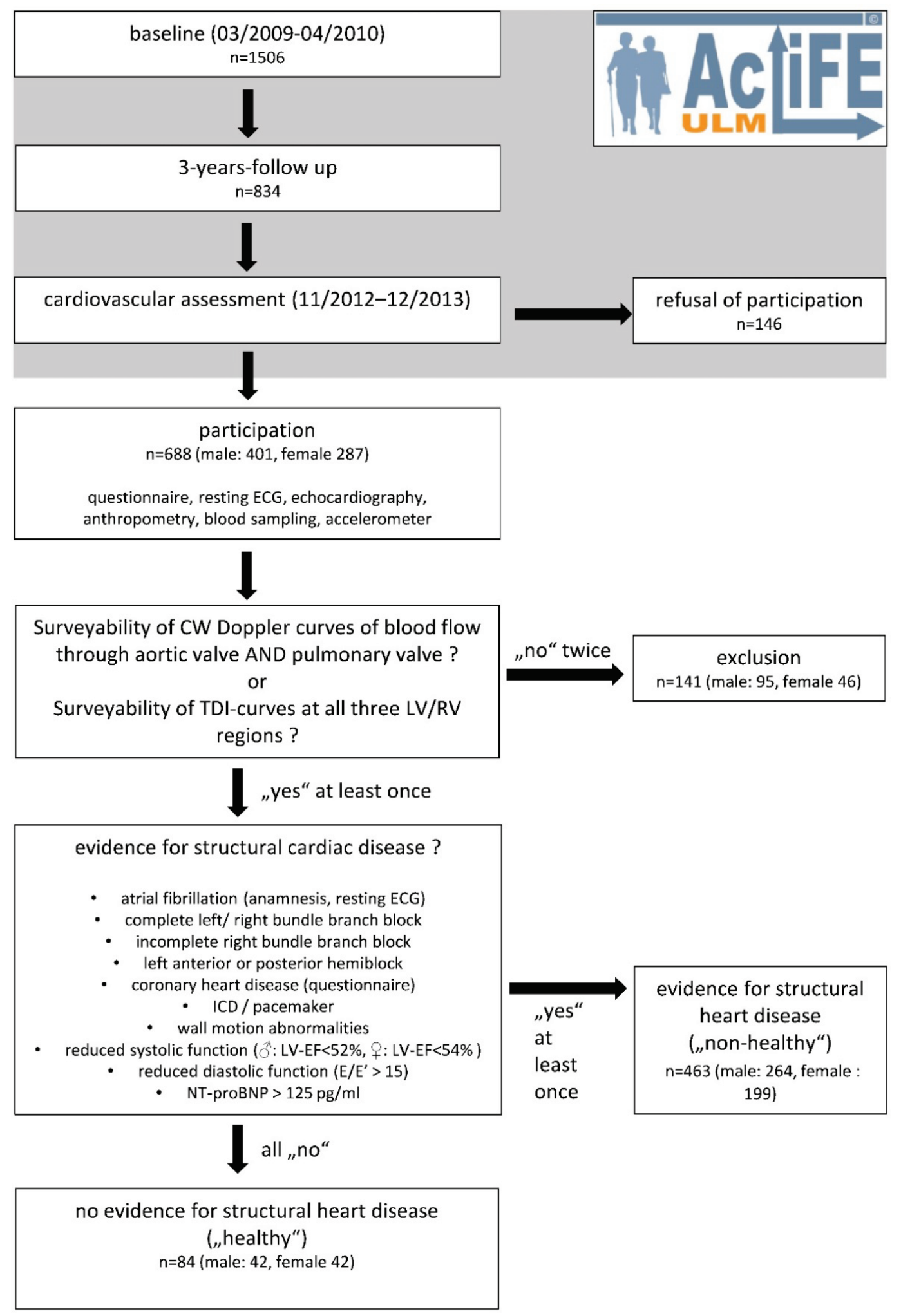

Fig. 1. Summary of inclusion/exclusion procedure. 


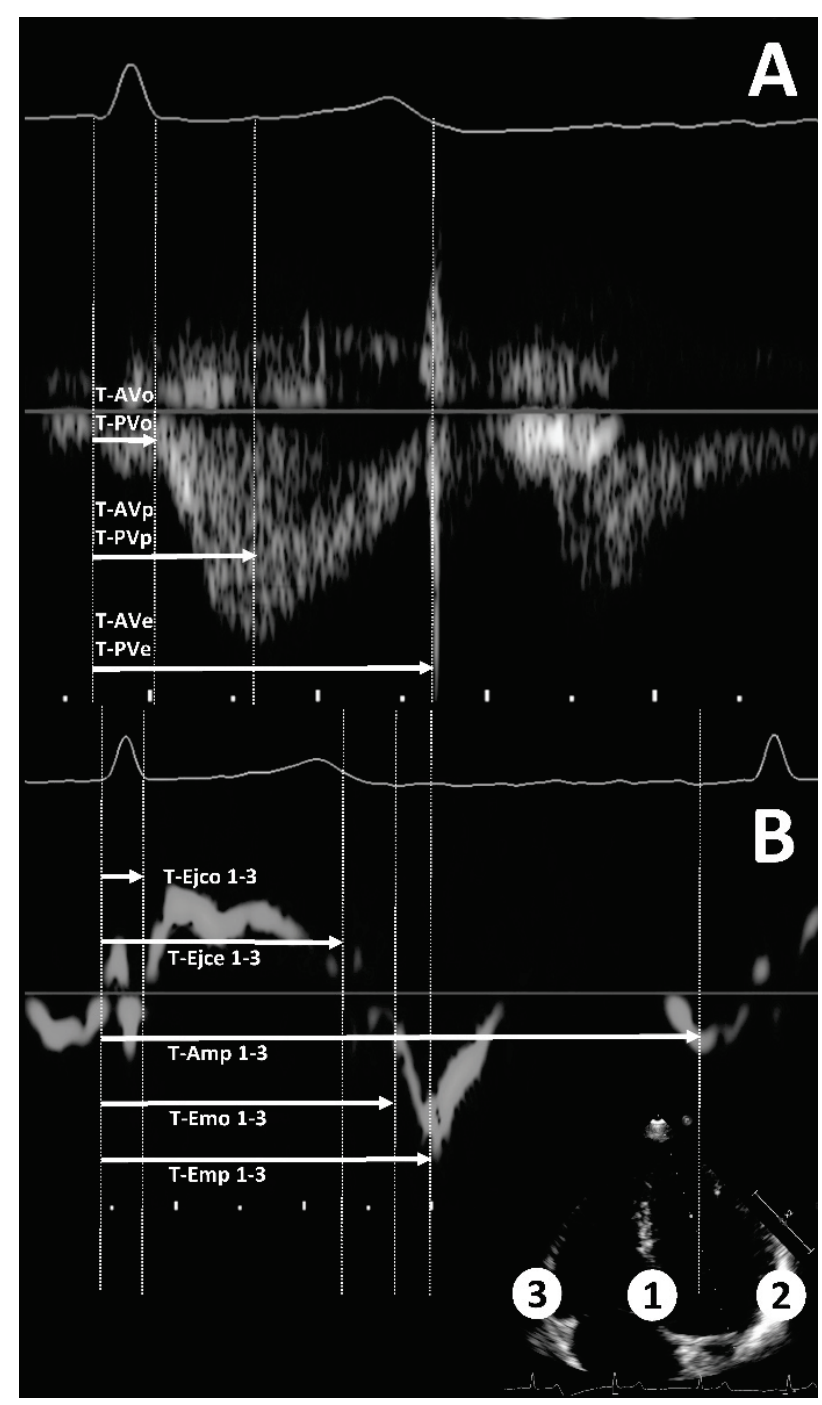

Fig. 2. (A) PW- and (B) TDI-curves with measured asynchrony parameters. $\mathrm{T}-\mathrm{AV} / \mathrm{p} / \mathrm{e}$ and $\mathrm{T}-\mathrm{PVo} / \mathrm{p} / \mathrm{e}$ : time from the beginning of QRS complex (bQRS) to onset (o), peak (p) and end (e) of

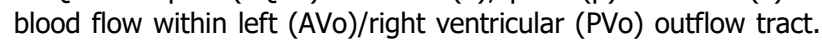
T-Ejco/e 1-3: time from bQRS complex to onset (o) and end (e) of ejection, measured at three different myocardial sites (see inset): 1 - interventricular septum, 2 - left ventricular free wall $1 \mathrm{~cm}$ blow mitral valve annulus, 3 - right ventricular free wall $1 \mathrm{~cm}$ below tricuspid valve annulus. T-Emo/p 1-3: time from bQRS complex to onset (o) and peak (p) of early diastolic myocardial velocity (Em) measured at sites 1-3. T-Amp 1-3: time from bQRS complex to peak (p) of late diastolic myocardial velocity $(\mathrm{Am})$ measured at sites $1-3$.

Synchrony analysis was performed offline. By using PW-Doppler curves (Fig. 2A), the time intervals from the beginning of the QRS-complex to the onset (T-AVo/T-PVo), peak (T-AVp, T-PVp) and end (T-AVe, $\mathrm{T}-\mathrm{PVe}$ ) were measured both for the left and the right ventricle (Linde et al. 2012, Yu et al. 2009). Concerning TDI measurements (Fig. 2B), the time interval from the beginning of the QRS complex to maximum velocity of the S-wave was classically used for evaluation of systolic asynchrony in cardiac resynchronization studies (Faber et al. 2003, Linde et al. 2012). However, a clear peaking of the S-wave is often hard to detect, especially in subjects with reduced systolic contraction velocities (Perez de Isla et al. 2005, Perez de Isla et al. 2008). This situation resulted in substantial measurement errors in those studies (Chung et al. 2008) and is at least partly held responsible for the still suboptimal response prediction in CRT-therapy ( $\mathrm{Yu}$ et al. 2009). As similar problems were also expected in our geriatric cohort, we decided to evaluate the time intervals from the beginning of the QRS complex to the beginning (T-EjctoX) and the end (T-EjcteX) of ejection as done in other studies (Linde et al. 2012, Perez de Isla et al. 2005, Perez de Isla et al. 2008). For the evaluation of diastolic synchrony, the time intervals from the beginning of the QRS complex to the beginning (T-EmoX) and peak (T-EmpX) of the Em-wave and, respectively, peak (T-AmpX) of the Am-wave were measured in every left/right ventricular area. As the duration of ventricular diastole depends on the heart rate, all diastolic measurements were normalized to the heart rate at the moment of registration of the respective TDI curve (Quan et al. 2012, Schuster et al. 2005, Yu et al. 2007). By using these measurements, the actual parameters for the subject-specific evaluation of systolic and diastolic (early (analyses of Em) and late (analyses of Am)) LV/RV inter-, LV intra- and $\mathrm{RV}$ intraventricular synchrony were calculated (Faber et al. 2003, Linde et al. 2012). Table 1 gives an overview of the performed calculations.

To the best of our knowledge, no clear "normal values" for cardiac (a)synchrony in healthy subjects exist. In order to evaluate relevancy of asynchrony two provisional approaches in terms of model-like considerations were chosen. First (definition 1), calculated synchrony parameters were compared with established echocardiographic parameters used as a part of medical indication for cardiac resynchronization therapy. Here, a cut-off of $40 \mathrm{~ms}$ (Yu et al. 2009) is suggested for PW-Doppler based evaluation (equivalent in our study to the clinically-used parameter: InterSys-1) of potential interventricular asynchrony. InterSys-2-4 are not used clinically but again, a cut-off of $40 \mathrm{~ms}$ was assumed virtually. Concerning systolic TDI parameters, a cut-off of $65 \mathrm{~ms}$ ( $\mathrm{Yu}$ et al. 2009) for septal to lateral delay is used clinically and this cut-off value was provisionally transferred also to the other systolic TDI synchrony markers. To the best of our knowledge, no cut-off values for the diastolic TDI parameters have 
been defined. Second (definition 2), both in the healthy and non-healthy group, systolic or diastolic asynchrony was defined to be potentially relevant if in a subject a respective parameter exceeded a cut-off defined as mean value of the healthy group \pm 2 standard deviations.

Table 1. Calculated measures for synchrony. See Figure 2 for abbreviations of the measured parameters of synchrony.

\section{Calculation}

\begin{tabular}{|c|c|}
\hline \multicolumn{2}{|c|}{ Systolic asynchrony } \\
\hline \multicolumn{2}{|c|}{ Interventricular asynchrony } \\
\hline InterSys-1 & $|(T-A V o)-(T-P V o)|$ \\
\hline InterSys-2 & $|(T-A V p)-(T-P V p)|$ \\
\hline InterSys-3 & $|(T-A V e)-(T-P V e)|$ \\
\hline InterSys-4 & $|(T-A V e-T-A V o)-(T-P V e-T-P V o)|$ \\
\hline InterSys-5 & $|(\mathrm{T}-\mathrm{Ej} \operatorname{co} 2)-(\mathrm{T}-\mathrm{Ej} \operatorname{co} 3)|$ \\
\hline InterSys-6 & $|(T-E j c e 2)-(T-E j c e 3)|$ \\
\hline \multicolumn{2}{|c|}{ LV intraventricular asynchrony* } \\
\hline$L V$-IntraSys-1 & $|(T-E j c o 1)-(T-E j c o 2)|$ \\
\hline$L V$-IntraSys-2 & $|(T-E j c e 1)-(T-E j c e 2)|$ \\
\hline \multicolumn{2}{|c|}{$R V$ intraventricular asynchrony } \\
\hline$R V$-IntraSys-1 & $|(T-E j c o 1)-(T-E j c o 3)|$ \\
\hline$R V$-IntraSys-2 & $|(T-E j c e 1)-(T-E j c e 3)|$ \\
\hline \multicolumn{2}{|c|}{ Diastolic asynchrony } \\
\hline \multicolumn{2}{|c|}{ Interventricular asynchrony } \\
\hline InterDia-1 & $\left|\left(\mathrm{T}-\mathrm{Emo} 2_{\mathrm{c}}\right)-\left(\mathrm{T}-\mathrm{Emo} 3_{\mathrm{c}}\right)\right|$ \\
\hline InterDia-2 & $\left|\left(\mathrm{T}-\mathrm{Emp} 2_{\mathrm{c}}\right)-\left(\mathrm{T}-\mathrm{Emp} 3_{\mathrm{c}}\right)\right|$ \\
\hline InterDia-3 & $\left|\left(\mathrm{T}-\mathrm{Amp} 2_{\mathrm{c}}\right)-\left(\mathrm{T}-\mathrm{Amp} 3_{\mathrm{c}}\right)\right|$ \\
\hline \multicolumn{2}{|c|}{ LV intraventricular asynchrony } \\
\hline$L V$-IntraDia-1 & $\left|\left(\mathrm{T}-\mathrm{Emo1} 1_{\mathrm{c}}\right)-\left(\mathrm{T}-\mathrm{Emo} 2_{\mathrm{c}}\right)\right|$ \\
\hline$L V$-IntraDia-2 & $\left|\left(\mathrm{T}-\mathrm{Emp} 1_{\mathrm{c}}\right)-\left(\mathrm{T}-\mathrm{Emp} 2_{\mathrm{c}}\right)\right|$ \\
\hline LV-IntraDia-3 & $\left|\left(\mathrm{T}-\mathrm{Amp} 1_{\mathrm{c}}\right)-\left(\mathrm{T}-\mathrm{Amp} 2_{\mathrm{c}}\right)\right|$ \\
\hline \multicolumn{2}{|c|}{$R V$ intraventricular asynchrony } \\
\hline$R V$-IntraDia-1 & $\left|\left(\mathrm{T}-\mathrm{Emo} 1_{\mathrm{c}}\right)-\left(\mathrm{T}-\mathrm{Emo} 3_{\mathrm{c}}\right)\right|$ \\
\hline$R V$-IntraDia-2 & $\left|\left(T-E m p 1_{c}\right)-\left(T-E m p 3_{c}\right)\right|$ \\
\hline$R V$-IntraDia-3 & $\left|\left(\mathrm{T}-\mathrm{Am} 12_{\mathrm{c}}\right)-\left(\mathrm{T}-\mathrm{Amp} 3_{\mathrm{c}}\right)\right|$ \\
\hline
\end{tabular}

c- corrected for heart rate: measure $* 1000 /$ cycle length [ms].

Reproducibility of echocardiographic asynchrony parameters is known to be problematic (Fraser et al. 2003, Mandysova et al. 2008, Vinereanu et al. 1999). To assess intraobserver variability of systolic parameters, T-AVo, T-PVo, T-Ejcto-1 and T-Ejcto-2 and consecutively InterSys-1 and LV-IntraSys-1 were assessed twice in all subjects of the subgroup of healthy people. In order to also evaluate reproducibility in non-healthy subgroup, T-Emp $1_{c}$ and T-Emp $2_{c}$ and consecutively LV-IntraDia-2 were also assessed twice in randomly selected $10 \%$ of all subjects of this group. These two procedures were done accordingly by a second observer for evaluation of interobserver variability. Time interval between the two assessments for intraobserver variability was $>1$ year. Based on these data, inter- and intraobserver variability have been evaluated with the following statistical approaches: 1) comparison of mean value (paired t-test), 2) intraclass correlation coefficient (ICC, two-way mixed model, absolute agreement) (Gisev et al. 2013), 3) coefficient of variance (COV) calculated as a percentage: standard deviation of the difference multiplied by 100 and divided by mean value of the two measurements (Synek 2008), and 4) BlandAltman analysis (Giavarina 2015).

SPSS 21 was used for statistical analysis. Data are presented as means with standard deviation and $95 \%$ confidence interval. Normality of the data was verified with the Shapiro-Wilk test. The continuous variables did show normal distribution and therefore, (paired) Student's t-test or ANOVA/least significant differenceBonferroni test were used for group comparison. Finally, statistical differences of nominal data were examined using Fisher's exact test. Two-sided p-values $<0.05$ were considered to be statistically significant.

\section{Results}

Table 2 gives an overview of the subgroup's characteristics including clinical, anthropometric and PA data, echo quality and standard echocardiographic parameters.

\section{Persons without evidence for structural heart disease}

Evaluation of interventricular systolic and diastolic synchrony

Measured PW-Doppler parameters of systolic interventricular synchrony of healthy persons (i.e. without evidence for structural heart disease according to our definition, see Methods) are given in Table 3A. On average, the onset of left ventricular ejection was slightly earlier than that of the right ventricle. Time to peak ejection velocity was also reached earlier in the left ventricle. The end of left ventricular ejection was also earlier by tendency. Total duration of ejection did not differ between both ventricles. 


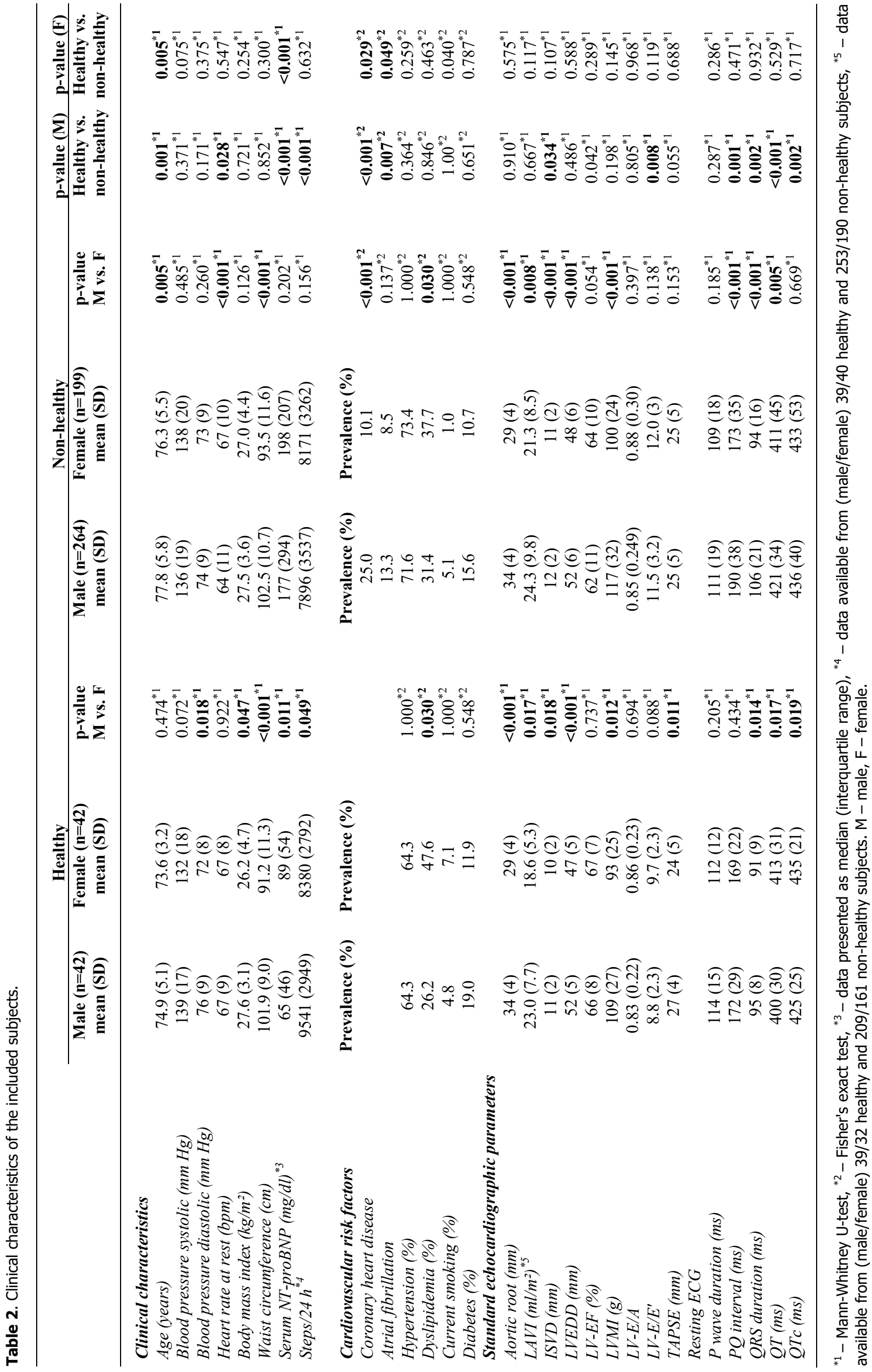




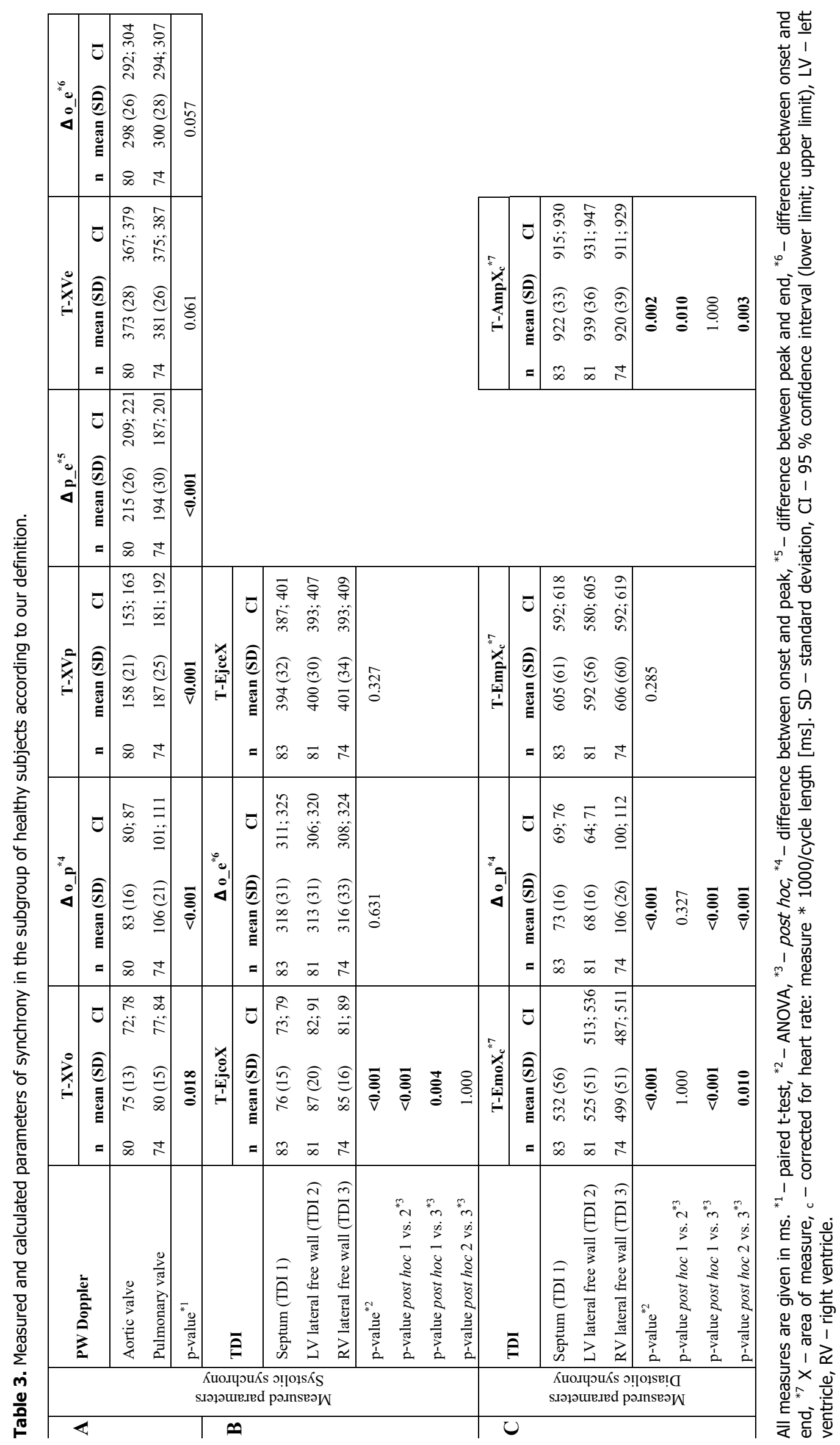


Table 4. Calculated parameters for systolic and diastolic asynchrony in healthy and non-healthy subjects.

\begin{tabular}{|c|c|c|c|c|c|c|c|}
\hline & \multicolumn{3}{|c|}{ Healthy } & \multicolumn{3}{|c|}{ Non-healthy } & \multirow{2}{*}{ p-value ${ }^{* 1}$} \\
\hline & n & mean (SD) & CI $(5 ; 95)$ & $\mathbf{n}$ & mean (SD) & CI $(5 ; 95)$ & \\
\hline \multicolumn{8}{|c|}{ Systolic asynchrony ${ }^{* 2}$} \\
\hline \multicolumn{8}{|c|}{ A. Interventricular asynchrony } \\
\hline InterSys-1 & 70 & $13(11)$ & $11 ; 16$ & 386 & $17(16)$ & $15 ; 19$ & 0.148 \\
\hline InterSys-2 & 70 & $34(22)$ & $28 ; 39$ & 386 & $37(26)$ & $34 ; 39$ & 0.510 \\
\hline InterSys-3 & 70 & $16(12)$ & $13 ; 19$ & 386 & $20(18)$ & $18 ; 22$ & 0.200 \\
\hline InterSys-4 & 70 & $17(11)$ & $14 ; 20$ & 386 & $20(17)$ & $18 ; 22$ & 0.394 \\
\hline InterSys-5 & 71 & $16(12)$ & $14 ; 19$ & 289 & $21(18)$ & $18 ; 23$ & 0.116 \\
\hline InterSys-6 & 71 & $20(17)$ & $17 ; 24$ & 289 & $23(19)$ & $21 ; 25$ & 0.342 \\
\hline \multicolumn{8}{|c|}{ B. $L V$ intraventricular asynchrony } \\
\hline$L V$-IntraSys-1 & 80 & $16(13)$ & $13 ; 19$ & 305 & $18(18)$ & $16 ; 20$ & 0.873 \\
\hline LV-IntraSys-2 & 80 & $14(12)$ & $11 ; 16$ & 305 & $17(14)$ & $15 ; 18$ & 0.132 \\
\hline \multicolumn{8}{|c|}{ C. $R V$ intraventricular asynchrony } \\
\hline$R V$-IntraSys-1 & 72 & $12(9)$ & $10 ; 15$ & 342 & $15(12)$ & $14 ; 16$ & 0.330 \\
\hline$R V$-IntraSys-2 & 72 & $21(16)$ & $17 ; 25$ & 342 & $22(17)$ & $20 ; 23$ & 0.753 \\
\hline \multicolumn{8}{|c|}{ Diastolic asynchrony $^{* 2}$} \\
\hline \multicolumn{8}{|c|}{ D. Interventricular asynchrony } \\
\hline InterDia-1 & 71 & $38(29)$ & $31 ; 45$ & 289 & $47(35)$ & $43 ; 51$ & 0.053 \\
\hline InterDia-2 & 71 & $33(28)$ & $27 ; 40$ & 289 & $43(34)$ & $39 ; 47$ & 0.020 \\
\hline InterDia-3 & 71 & $32(30)$ & $25 ; 39$ & 289 & $39(33)$ & $35 ; 43$ & 0.075 \\
\hline \multicolumn{8}{|c|}{ E. LV intraventricular asynchrony } \\
\hline LV-IntraDia-1 & 80 & $23(21)$ & $19 ; 28$ & 305 & $36(32)$ & $32 ; 40$ & 0.002 \\
\hline$L V$-IntraDia-2 & 80 & $24(21)$ & $19 ; 28$ & 305 & $35(34)$ & $31 ; 38$ & 0.020 \\
\hline$L V$-IntraDia-3 & 80 & $27(22)$ & $22 ; 32$ & 305 & $34(37)$ & $30 ; 38$ & 0.109 \\
\hline \multicolumn{8}{|c|}{ F. $R V$ intraventricular asynchrony } \\
\hline$R V$-IntraDia- 1 & 73 & $45(28)$ & $39 ; 52$ & 342 & $45(28)$ & $39 ; 52$ & 0.803 \\
\hline$R V$-IntraDia-2 & 73 & $36(27)$ & $30 ; 42$ & 342 & $39(32)$ & $36 ; 43$ & 0.692 \\
\hline$R V$-IntraDia-3 & 73 & $26(22)$ & $21 ; 31$ & 342 & $28(31)$ & $25 ; 32$ & 0.738 \\
\hline
\end{tabular}

SD - standard deviation, CI - $95 \%$ confidence interval (lower limit; upper limit). LV - left ventricle, RV - right ventricle. ${ }^{* 1}$ U-test. ${ }^{* 2}$ all measures in $\mathrm{ms}$.

The subject-specifically calculated PW-Doppler parameters for the evaluation of actual systolic interventricular synchrony are shown in Table 4A. Consistent with the above-mentioned averages of the measured parameters, InterSys1-3 speak for a minimal interventricular systolic asynchrony.

TDI curves assessed in area 2 and 3 (see Methods and Fig. 1) are usable for the evaluation of both systolic and diastolic interventricular synchrony. Contrarily to the PW-Doppler measurements, average time from the beginning of the QRS-complex to ejectiononset (T-Ejco) and ejection-end (T-Ejce) did not differ between basal right and left ventricular free wall (Table 3B). However, TDI parameters for interventricular synchrony (InterSys-5 and InterSys-6, Table 4A) also speak for a minimal interventricular systolic asynchrony.

Measured TDI parameters for evaluation of diastolic interventricular synchrony are presented in Table 3C. On average, left ventricular diastole began later $\left(\mathrm{T}-\mathrm{EmoX}_{\mathrm{c}}\right)$ than in the right ventricle, whereas peaks of early diastolic velocities $\left(\mathrm{T}-\mathrm{EmpX} \mathrm{X}_{\mathrm{c}}\right)$ occurred simultaneously. Peak velocity of atrial enddiastolic contraction (T-AmpX $\mathrm{X}_{\mathrm{c}}$ ) was also reached later in the left than in the right ventricle. The calculated parameters for the actual evaluation of interventricular diastolic synchrony (Table 4A) pointed to a minimally asynchronous beginning of early/late diastolic velocity increase (InterDia-1 and InterDia-3) and also peaking of early diastolic velocities (InterDia-2). 
Evaluation of left ventricular intraventricular systolic and diastolic synchrony

TDI curves or area 1 and 2 (Fig. 1) were used for evaluating the left ventricular intraventricular systolic and diastolic synchrony. On average, ejection onset of interventricular septum was earlier than that of left ventricular free wall, whereas ejection ended simultaneously (Table 3B). According to the calculated parameters (LV-IntraSys-1 and LV-IntraSys-2, Table 4B), a minimal left ventricular intraventricular systolic asynchrony was observed.

Concerning the diastole, on average, no differences of the beginning of increase and peaking of early diastolic velocities (T-EmoX $\mathrm{X}_{\mathrm{c}}$ and $\mathrm{T}-\mathrm{EmpX} \mathrm{X}_{\mathrm{c}}$, Table 3C) within interventricular septum and the left ventricular free wall were detectable. Contrarily, the peak of late atrial velocity was reached earlier within the interventricular septum than in the left ventricular free wall (T-AmpX $\mathrm{X}_{\mathrm{c}}$, Table 3C) on average.

Calculated parameters (LV-IntraDia-1-3, Table 4E) indicated a minimal intraventricular asynchrony of the early and late left ventricular diastole.

Evaluation of right ventricular intraventricular systolic and diastolic synchrony

Finally, right ventricular intraventricular systolic and diastolic synchrony was evaluated by analyzing the TDI curves of area 1 and 3 (Fig. 1). Comparable to the left ventricle, the onset of ejection was detectable earlier in the interventricular septum than in the right ventricular free wall, whereas it ended simultaneously (Table 3B) on average. Calculated parameters pointed to a minimal (RV-IntraSys-1 and RV-IntraSys-2, Table 4C) right ventricular systolic interventricular asynchrony.

Differently to the left ventricle, on average, the beginning of the increase of early diastolic velocity within the interventricular septum was earlier than within the basal right ventricular free wall, while its peaking occurred simultaneously just like the peaking of late enddiastolic velocity did (Table 3B). Calculated measures (RV-InterDia-1-3) speak for a minimal right ventricular interventricular diastolic asynchrony (Table 4F).

Influence of sex and QRS duration and relevancy of asynchrony

T-PVo (M: $79 \pm 16 \mathrm{~ms}$ vs. F: $83 \pm 15 \mathrm{~ms}$, $\mathrm{p}=0.021)$, T-PVe (M: $376 \pm 25 \mathrm{~ms}$ vs. F: $386 \pm 26 \mathrm{~ms}$, $\mathrm{p}=0.005$ ), T-Ejce1 (M: $383 \pm 31 \mathrm{~ms}$ vs. F: $404 \pm 29 \mathrm{~ms}$, $\mathrm{p}=0.002$ ), T-Ejce2 (M: $390 \pm 30 \mathrm{~ms}$ vs. F: $410 \pm 28 \mathrm{~ms}$, $\mathrm{p}=0.005), \mathrm{T}-\mathrm{Emo3}_{\mathrm{c}}$ (M: $477 \pm 50 \mathrm{~ms}$ vs. F: $523 \pm 42 \mathrm{~ms}$, $\mathrm{p}<0.001$ ) and T-Emp $3_{\mathrm{c}}$ (M: $586 \pm 61 \mathrm{~ms}$ vs. F: $626 \pm 53 \mathrm{~ms}$, $\mathrm{p}=0.008$ ) slightly differed between male and female persons. However, none of the calculated asynchrony parameters showed significant sex differences. Only LV-IntraSys-2 did show a small but significant correlation with QRS duration $(\mathrm{r}=0.26, \mathrm{p}=0.020)$.

Table 5 gives an overview of the amount of healthy subjects fulfilling the respective criterions of asynchrony according to our definitions (see Methods). With the exception of InterSys-2, less than $5 \%$ of all healthy subjects met a respective criterion according to definition 1 (Table 5A). Concerning definition 2 (Table 5B), also only a small amount of subjects (at most $8.2 \%$ depending on the respective parameter) exhibited relevant asynchrony.

\section{Asynchrony in subjects with evidence for structural heart disease}

To get an idea of potential effects of structural heart disease on cardiac synchrony in the elderly, the hitherto presented analyses were again performed in the subgroup of non-healthy subjects according to our definition (see Methods). For reasons of clarity, only the calculated variables of asynchrony are presented (Table 4). No significant differences of all calculated parameters of systolic interventricular asynchrony, LV intraventricular asynchrony and $\mathrm{RV}$ intraventricular asynchrony were observed (Table 4A-C). InterDia-1-3 pointed to an increase of diastolic interventricular asynchrony in comparison to healthy subjects at least by tendency (Table 4D). LV-IntraDia-1-3 gave evidence for a significant increase of early but not late diastolic LV intraventricular asynchrony as a consequence of structural heart disease (Table 4E). Minimal diastolic $\mathrm{RV}$ intraventricular asynchrony did not differ between the healthy and non-healthy group (Table 4F).

InterSys-2 (M: $35 \pm 25 \mathrm{~ms}$ vs. F: $41 \pm 30 \mathrm{~ms}$, $\mathrm{p}=0.018$ ), InterSys-5 (M: $-1 \pm 26 \mathrm{~ms}$ vs. F: $8 \pm 25 \mathrm{~ms}$, $\mathrm{p}=0.003$ ) and RV-IntraSys-1 (M: $16 \pm 14 \mathrm{~ms}$ vs. F: $13 \pm 10 \mathrm{~ms}, \mathrm{p}=0.013$ ) revealed minimal but significant sex-differences. InterSys- $1(\mathrm{r}=0.35, \mathrm{p}<0.001)$, InterSys-3 $(\mathrm{r}=0.30, \mathrm{p}<0.001)$ and RV-IntraSys- $1(\mathrm{r}=0.28, \mathrm{p}<0.001)$ slightly correlated with QRS duration. 


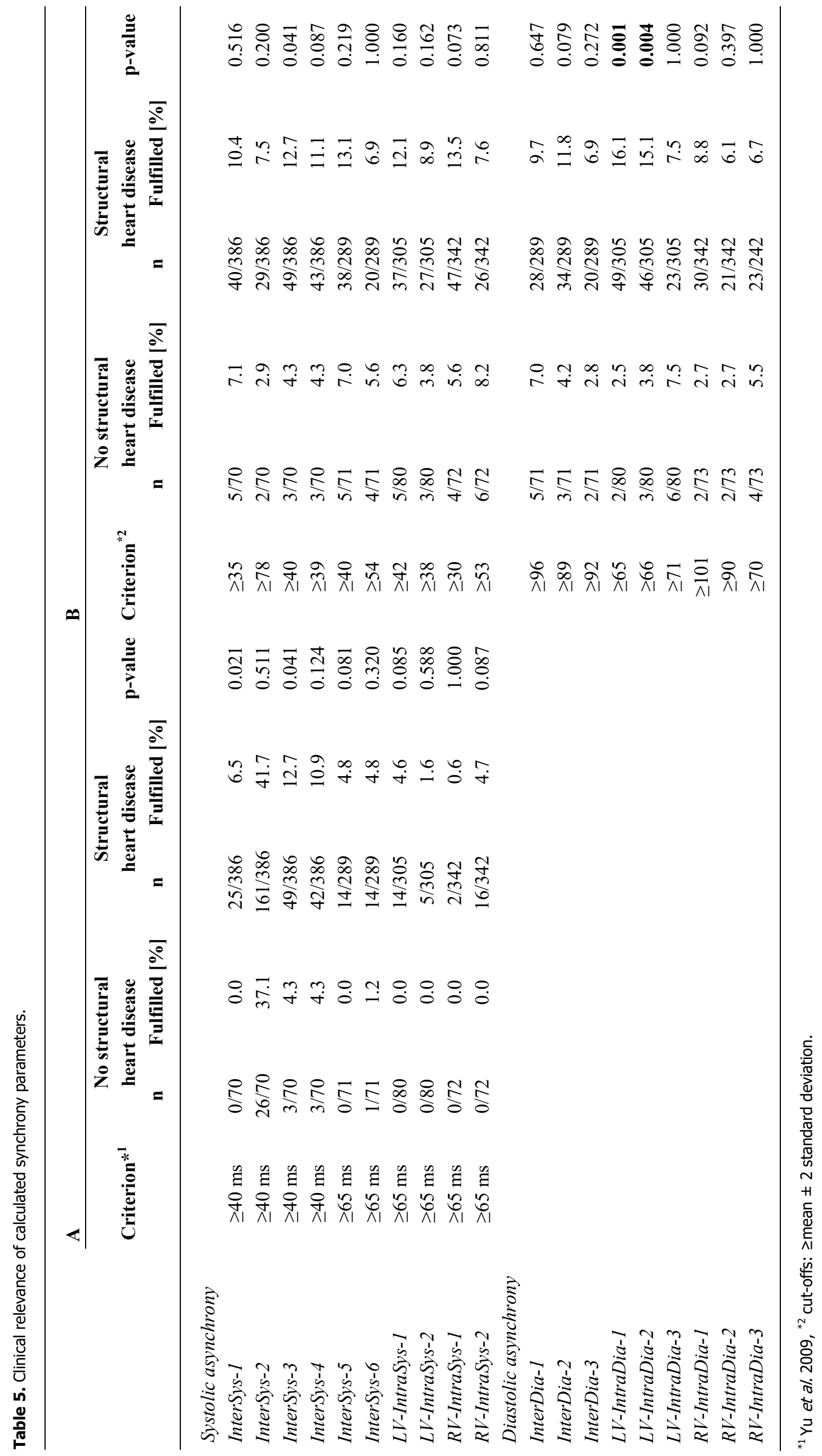




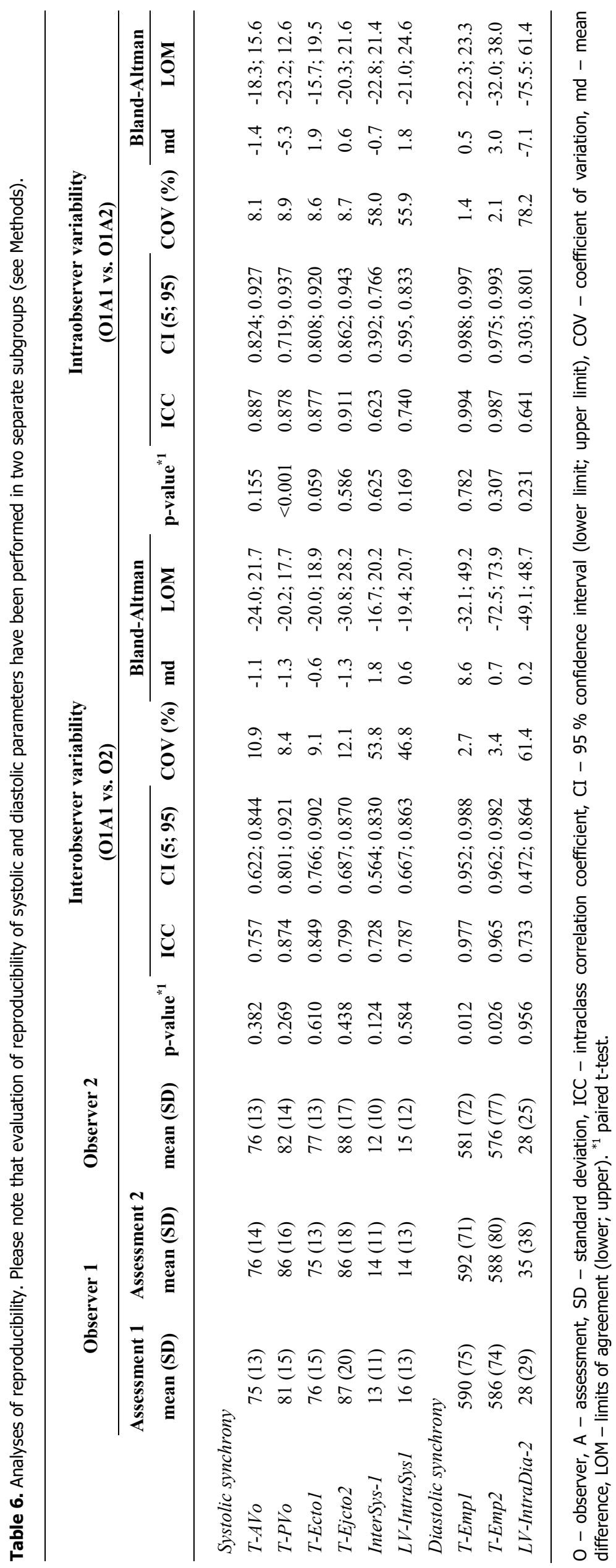


Percentage of non-healthy persons fulfilling a respective literature criterion did not differ from the percentage of healthy persons (Table 5A). A similar situation was also observed concerning our analyses in which our self-defined (definition 2, see Methods) cut-off values for systolic asynchrony were used (Table 5B). However, in good concordance with the above mentioned significant differences of LV-IntraDia-1 and LV-IntraDia-2, cut-off values of these parameters for early LV intraventricular diastolic asynchrony were significantly exceeded by more often by non-healthy than by healthy subjects.

\section{Inter- and intraobserver variability}

Analyses of reproducibility are presented in Table 6. Concerning interobserver variability, mean values of all exemplarily evaluated systolic parameters did not differ statistically, whereas means of the measured but not calculated diastolic parameters of observer 2 were slightly smaller than in observer 1. Intraclass correlation coefficients (COV) revealed mostly barely acceptable (COV 0.7-0.8) to good (COV 0.8-0.9; measured systolic variables) and excellent (COV $>0.9$; measured diastolic variables) interrater variability. However, COVs of the calculated variables were consecutively lower with a large confidence interval including inacceptable values $(\mathrm{COV}<0.7)$ indicating problematic reproducibility of theses variables in the same way as the comparably high limits of agreements (LOM) of the Bland-Altman analyses. Similar results were found for intraobserver variability as also shown in Table 6.

\section{Discussion}

In our cross-sectional study, synchrony of cardiac contraction was evaluated in a population-based geriatric cohort. Both in the subgroups of healthy and non-healthy subjects according to our definitions, mean values of our calculated parameters of synchrony were generally small and only a minority of subjects fulfilled defined asynchrony criteria. This suggests that both systolic and diastolic asynchrony of contraction were not relevant in our cohort. Within the subgroup of non-healthy subjects, most variables of diastolic interand LV intraventricular asynchrony were slightly increased in comparison to the subgroup of healthy subjects indicating that structural heart diseases might go along with an increase of diastolic asynchrony.
For the purpose of the study we used strict criteria to define "healthy" persons without evidence for structural heart disease. NT-proBNP was an important parameter using the recommended cut-off of $125 \mathrm{pg} / \mathrm{ml}$ for diagnosis of heart failure in a non-acute setting (McMurray et al. 2012). In this way, any hemodynamically relevant valvular heart diseases (Bergler-Klein et al. 2014, Moura et al. 2008, Troughton et al. 2009) and diseases of pulmonary circulation (Galie et al. 2016) were adequately ruled out. We did not exclude subjects with arterial hypertension or diabetes mellitus per se. For example, the diagnosis "atrial hypertension" according to our definition subsumes subjects from both extremes in terms of properly treated arterial hypertension without any secondary complications on the one side and longstanding, untreated hypertensive subjects with severe secondary cardiac structural alteration on the other side. The same applies to diabetes mellitus and diabetic cardiomyopathy. The final common pathway of left atrial volume and/or pressure overload caused by these two diseases but also by other various factors in an individual subject is an severity-dependent increased release of BNP (Mahadavan et al. 2014). Therefore, hypertensive/diabetic cardiomyopathy (as a potential consequence of insufficiently treated underlying disease which may affect echocardiographic measures) were also adequately ruled out by normal NT-proBNP (BerglerKlein et al. 2014, Galie et al. 2016, Moura et al. 2008, Santos et al. 2014, Troughton and Richards 2009). As a result of our strict criteria, about $88 \%$ of all subjects from our initial cohort were pooled in the non-healthy subgroup.

A relevant amount of the absolute value of our measured parameters represents myocardial conduction of excitation. The longer systolic TDI time intervals of both the left and right ventricular free wall in comparison to the interventricular septum with close proximity to the specific cardiac conduction system (AV node) were in line with these findings. As cardiac size differs between men and women (Lang et al. 2015), one might also expect differences in measured parameters (males $>$ females) due to relatively longer conduction times in larger hearts. However, almost none of the measured parameters exhibited significant sex differences and if they did, time intervals were longer in female subjects. This might be explained by known sex-specific differences of EC-coupling (Parks et al. 2013). Ultimately, these differences had no consequence for the actual evaluation of cardiac synchrony. Almost none of the calculated parameters of asynchrony correlated with 
QRS duration which is rather not surprising as QRS duration was normal by definition within this subgroup. As EC-coupling is also affected by cardiac afterload (Janssen 2010), a potential dependency of our evaluated asynchrony parameters and blood pressure at the time of echocardiographic measurement might exist. However, we were not able to evaluate this relation appropriately in our cohort, as the range of the observed blood pressure values during echocardiography within our cohort was too small. Due to similar reasons, namely the limited age span of people $>65$ in our study, we also could not closer examine the potential association of asynchrony and age.

Studies evaluating synchrony of contraction in "healthy" subjects are scarce. To the best of our knowledge, our study is even the first one concerning a geriatric cohort. Quan et al. (2012) studied 88 "healthy subjects" ( $40 \pm 15$ years, $48 \%$ male). In accordance to our data, the authors also reported of earlier peaking of systolic outflow in the left (T-AVp) than in the right (T-PVp) ventricle with simultaneous ending. Contrarily, no difference of the beginning of systolic outflow between left and right ventricle was observed. Comprehensive systolic and diastolic asynchrony TDI data was presented by Yu et al. (2003) in a study which included 106 ,healthy subjects" (64.3 \pm 9.5 years, $60 \%$ male). Here, time to isovolumic contraction and time to peak systolic contraction (as a difference to T-Ejcto and T-Ejce in our study) and time to peak early and late diastolic velocities, respectively, (equivalent to T-EmpX and T-Ampx) were measured, whereby no correction for heart rate of the diastolic measures was used. The authors found that systole and diastole within the left ventricle were "highly synchronized" but the time to isovolumic and peak systolic velocities of the right ventricle were - comparable to our data with reservations - slightly delayed. In a study by Sadeghian et al. (2010), the time to peak systolic velocity of basal septal and lateral left ventricle was measured in 65 "healthy young adult volunteers" (30.1 \pm 7.5 years, $52 \%$ male) and also septal to lateral delay (equivalent to LV-IntraSys-1 and LV-IntraSys-2 with reservations) was calculated (Sadeghian et al. 2010). Mean values of time to peak systolic velocities of basal septal and lateral ventricle differed by $\approx 45 \mathrm{~ms}$ and calculated mean septal to lateral delay was $55 \pm 39 \mathrm{~ms}$. A similar septal to lateral delay (median $66 \mathrm{~ms}$, by measuring time to peak systolic velocities as well) was reported by Miyazaki et al. (2008) in a study including 40 "healthy subjects" (age $47 \pm 15$ years, $60 \%$ male). So did $\mathrm{Ng}$ et al. (2008) in a further study $(58 \pm 40 \mathrm{~ms}, 122$ "healthy" subjects, $44 \pm 13$ years, $53 \%$ male). These results are contrary to ours with only a minimal left ventricular intraventricular systolic asynchrony. The mentioned methodical problems of measuring the time to peak velocity interval might be responsible for this fact. In the last study mentioned (Ng et al. 2008), also a septal to lateral delay of early peak diastolic velocity (equivalent to LV-IntraDia-1) of $15 \pm 15 \mathrm{~ms}$ was reported. This is in concordance to our results. Yu et al. (2007) evaluated left ventricular intraventricular systolic and diastolic asynchrony via TDI in "100 healthy volunteers recruited from the community" (64.2 \pm 9.4 years, $71 \%$ male) by using a twelve segmental model. No details concerning the time intervals from the beginning of QRS complex to peak systolic and, respectively, peak early myocardial velocities were given but a mean maximum difference of $54 \pm 23 \mathrm{~ms}$ (systolic) and $63 \pm 25 \mathrm{~ms}$ (diastolic) between the time intervals of the different segments indicated a left ventricular intraventricular systolic and diastolic asynchrony in healthy individuals. With reservations, both the systolic and diastolic extent of asynchrony seemed to be slightly bigger than in our study. Again, the use of the problematic systolic peak velocities might be an explanation for the discrepancy concerning systolic asynchrony. The differences concerning diastolic asynchrony remain unclear. By using a six segmental model of the left ventricle, a mean maximum difference (again no specific time intervals were reported) of time to peak systolic and early diastolic velocity of $12 \pm 10 \mathrm{~ms}$ and, respectively, $10 \pm 9 \mathrm{~ms}$ in a cohort of 35 "healthy adults" were reported by Wang et al. (2007). Therefore, again with the abovementioned reservations, extent of left ventricular intraventricular systolic and early diastolic asynchrony was comparable to our cohort with geriatric subjects.

To the best of our knowledge, no populationbased studies evaluating cardiac synchrony are available yet. In our subgroup with non-healthy subjects no differences of the evaluated systolic synchrony parameters in comparison to the subgroup with healthy subjects were observed. The percentage of subjects with potentially relevant systolic asynchrony according to our definitions also did not differ. However, extent of diastolic left ventricular intraventricular asynchrony and also - by tendency - diastolic interventricular asynchrony was increased in comparison to the healthy subgroup. $\mathrm{Yu}$ et al. (2007) reported of the presence of diastolic asynchrony in a cohort with heart failure patients despite narrow QRS-complex. As especially diastolic heart 
failure has a known increased prevalence in the elderly, our observations therefore seem to be conclusive.

The results of our analyses concerning reproducibility are the major limitation of our study. "Classical" echocardiographic synchrony markers obtained by PW- and TDI-Doppler were analyzed in our study. Comparable with other studies (Fraser et al. 2003, Mandysova et al. 2008, Vinereanu et al. 1999), particularly the reproducibility of the calculated variables was at least partly problematic so that our results have to be interpreted with caution. It is noteworthy but not apologetic that other similar studies (Miyazaki et al. 2008, Ng et al. 2008, Quan et al. 2012, Sadeghian et al. 2010, Wang et al. 2007, Yu et al. 2003, Yu et al. 2007) also did not pay adequate attention to this important issue. Meanwhile, imaging modalities like for example strain analysis (Gorcsan et al. 2012) with better reproducibility are available which will be included in echocardiographic data acquisition of the next follow-up of our cohort. As another limitation, it has to be noticed that our "non-healthy" group is extremely heterogeneous concerning clinical characteristics and therefore, this may hide some potentially significant differences of synchrony in particular non-healthy subgroups.

\section{Conclusion}

Despite known age-related alterations of cardiac conduction system and excitation-contraction coupling, only a minimal extent of asynchrony of contraction was found in our geriatric cohort both in the subgroups of healthy and non-healthy.

To conclude, no evidence that physiological ageing might go along with relevant left or right ventricular systolic or diastolic electromechanical asynchrony was found. However, our population-based data support the results from other studies with rather selected cohorts that structural heart diseases might go along with increased diastolic asynchrony.

\section{Conflict of Interest}

There is no conflict of interest.

\section{Acknowledgements}

With kind support of the team of Division of Sports and Rehabilitation Medicine, Ulm, Germany. The ActiFEUlm study was funded partly by a grant from the Ministry of Science, Research and Arts, State of BadenWuerttemberg, Germany and by own means.

\section{References}

BERGLER-KLEIN J, GYONGYOSI M, MAURER G: The role of biomarkers in valvular heart disease: focus on natriuretic peptides. Can J Cardiol 30: 1027-1034, 2014.

CARERJ S, RAFFA S, MARTINIELLO AR, LETO A, SCANDURA S, CAPASSO F, LA CARRUBBA S, CITRO R, IZZO A, MANGANO S, ZITO C, PENCO M, CASO P: Ventricular mechanical asynchrony in patients with different degrees of systolic dysfunction: results from AVE Registry by the Italian Society of Cardiovascular Echography (SIEC). Echocardiography 27: 110-116, 2009.

CHAN CP, ZHANG Q, YIP GW, FUNG JW, LAM YY, LEE PW, WU EB, SHANG Q, LIANG Y, YU CM: Relation of left ventricular systolic dyssynchrony in patients with heart failure to left ventricular ejection fraction and to QRS duration. Am J Cardiol 102: 602-605, 2008.

CHIAO YA, RABINOVITCH PS: The aging heart. Cold Spring Harb Perspect Med 5: a025148, 2015.

CHOW GV, MARINE JE, FLEG JL: Epidemiology of arrhythmias and conduction disorders in older adults. Clin Geriatr Med 28: 539-553, 2012.

CHUNG ES, LEON AR, TAVAZZI L, SUN JP, NIHOYANNOPOULOS P, MERLINO J, ABRAHAM WT, GHIO S, LECLERCQ C, BAX JJ, YU CM, GORCSAN J 3RD, ST JOHN SUTTON M, DE SUTTER J, MURILLO J: Results of the Predictors of Response to CRT (PROSPECT) trial. Circulation 117: 2608-2616, 2008.

DENKINGER MD, FRANKE S, RAPP K, WEINMAYR G, DURAN-TAULERIA E, NIKOLAUS T, PETER R: Accelerometer-based physical activity in a large observational cohort - study protocol and design of the activity and function of the elderly in Ulm (ActiFE Ulm) study. BMC Geriatr 10: 50, 2010.

FABER L, LAMP B, HERING D, BOGUNOVIC N, SCHOLTZ W, HEINTZE J, VOGT J, HORSTKOTTE D: Analysis of inter- and intraventricular asynchrony by tissue Doppler echocardiography (in German). Z Kardiol 92: 994-1002, 2003.

FERIDOONI HA, DIBB KM, HOWLETT SE: How cardiomyocyte excitation, calcium release and contraction become altered with age. J Mol Cell Cardiol 83: 62-72, 2015. 
FRASER AG, PAYNE N, MADLER CF, JANEROT-SJOBERG B, LIND B, GROCOTT-MASON RM, IONESCU AA, FLORESCU N, WILKENSHOFF U, LANCELLOTTI P, WUTTE M, BRODIN LA: Feasibility and reproducibility of off-line tissue Doppler measurement of regional myocardial function during dobutamine stress echocardiography. Eur J Echocardiogr 4: 43-53, 2003.

GALIE N, HUMBERT M, VACHIERY JL, GIBBS S, LANG I, TORBICKI A, SIMONNEAU G, PEACOCK A, VONK NOORDEGRAAF A, BEGHETTI M, GHOFRANI A, GOMEZ SANCHEZ MA, HANSMANN G, KLEPETKO W, LANCELLOTTI P, MATUCCI M, MCDONAGH T, PIERARD LA, TRINDADE PT, ZOMPATORI M, ET AL.: 2015 ESC/ERS Guidelines for the diagnosis and treatment of pulmonary hypertension: The Joint Task Force for the Diagnosis and Treatment of Pulmonary Hypertension of the European Society of Cardiology (ESC) and the European Respiratory Society (ERS): Endorsed by: Association for European Paediatric and Congenital Cardiology (AEPC), International Society for Heart and Lung Transplantation (ISHLT). Eur Heart J 37: 67-119, 2016.

GIAVARINA D: Understanding Bland Altman analysis. Biochem Med 25: 141-151, 2015.

GISEV N, BELL JS, CHEN TF: Interrater agreement and interrater reliability: key concepts, approaches, and applications. Res Social Adm Pharm 9: 330-338, 2013.

GORCSAN J 3RD, MAREK JJ, ONISHI T: The contemporary role of echocardiography in improving patient response to cardiac resynchronization therapy. Curr Cardiovasc Imaging Rep 5: 462-472, 2012.

JANSSEN PM: Myocardial contraction-relaxation coupling. Am J Physiol 299: H1741-H1749, 2010.

KARAVIDAS A, LAZAROS G, TSIACHRIS D, PYRGAKIS V: Aging and the cardiovascular system. Hellenic $J$ Cardiol 51: 421-427, 2010.

LAFITTE S, BORDACHAR P, LAFITTE M, GARRIGUE S, REUTER S, REANT P, SERRI K, LEBOUFFOS V, BERRHOUET M, JAIS P, HAISSAGUERRE M, CLEMENTY J, ROUDAUT R, DEMARIA AN: Dynamic ventricular dyssynchrony: an exercise-echocardiography study. $J$ Am Coll Cardiol 47: 2253-2259, 2006.

LANG RM, BADANO LP, MOR-AVI V, AFILALO J, ARMSTRONG A, ERNANDE L, FLACHSKAMPF FA, FOSTER E, GOLDSTEIN SA, KUZNETSOVA T, LANCELLOTTI P, MURARU D, PICARD MH, RIETZSCHEL ER, RUDSKI L, SPENCER KT, TSANG W, VOIGT JU: Recommendations for cardiac chamber quantification by echocardiography in adults: an update from the American Society of Echocardiography and the European Association of Cardiovascular Imaging. $J$ Am Soc Echocardiogr 28: 1-39.e14, 2015.

LINDE C, ELLENBOGEN K, MCALISTER FA: Cardiac resynchronization therapy (CRT): clinical trials, guidelines, and target populations. Heart Rhythm 9: S3-S13, 2012.

MAHADAVAN G, NGUYEN TH, HOROWITZ JD: Brain natriuretic peptide: a biomarker for all cardiac disease? Curr Opin Cardiol 29: 160-166, 2014.

MANDYSOVA E, MRAZ T, TABORSKY M, NIEDERLE P: Reproducibility of tissue Doppler parameters of asynchrony in patients with advanced LV dysfunction. Eur J Echocardiogr 9: 509-515, 2008.

MCMURRAY JJ, ADAMOPOULOS S, ANKER SD, AURICCHIO A, BOHM M, DICKSTEIN K, FALK V, FILIPPATOS G, FONSECA C, GOMEZ-SANCHEZ MA, JAARSMA T, KOBER L, LIP GY, MAGGIONI AP, PARKHOMENKO A, PIESKE BM, POPESCU BA, RONNEVIK PK, RUTTEN FH, SCHWITTER J, ET AL.: ESC guidelines for the diagnosis and treatment of acute and chronic heart failure 2012: The Task Force for the Diagnosis and Treatment of Acute and Chronic Heart Failure 2012 of the European Society of Cardiology. Developed in collaboration with the Heart Failure Association (HFA) of the ESC. Eur J Heart Fail 14: 803-869, 2012.

MIRZA M, STRUNETS A, SHEN WK, JAHANGIR A: Mechanisms of arrhythmias and conduction disorders in older adults. Clin Geriatr Med 28: 555-573, 2012.

MIYAZAKI C, POWELL BD, BRUCE CJ, ESPINOSA RE, REDFIELD MM, MILLER FA, HAYES DL, CHA YM, OH JK: Comparison of echocardiographic dyssynchrony assessment by tissue velocity and strain imaging in subjects with or without systolic dysfunction and with or without left bundle-branch block. Circulation 117: 2617-2625, 2008.

MOURA LM, ROCHA-GONCALVES F, ZAMORANO JL, BARROS I, BETTENCOURT P, RAJAMANNAN N: New cardiovascular biomarkers: clinical implications in patients with valvular heart disease. Expert Rev Cardiovasc Ther 6: 945-954, 2008. 
NAGUEH SF, APPLETON CP, GILLEBERT TC, MARINO PN, OH JK, SMISETH OA, WAGGONER AD, FLACHSKAMPF FA, PELLIKKA PA, EVANGELISA A: Recommendations for the evaluation of left ventricular diastolic function by echocardiography. Eur J Echocardiogr 10: 165-193, 2009.

NG AC, TRAN DA T, NEWMAN M, ALLMAN C, VIDAIC J, LO ST, HOPKINS AP, LEUNG DY: Left ventricular longitudinal and radial synchrony and their determinants in healthy subjects. $J$ Am Soc Echocardiogr 21: 1042-1048, 2008.

PARKS RJ, HOWLETT SE: Sex differences in mechanisms of cardiac excitation-contraction coupling. Pflugers Arch 465: 747-763, 2013.

PEREZ DE ISLA L, FLORIT J, GARCIA-FERNANDEZ MA, EVANGELISTA A, ZAMORANO J: Prevalence of echocardiographically detected ventricular asynchrony in patients with left ventricular systolic dysfunction. $J$ Am Soc Echocardiogr 18: 850-859, 2005.

PEREZ DE ISLA L, PORRO R, PARE JC, DE LA MORENA G, MACAYA C, ZAMORANO J: Relationship between intraventricular cardiac asynchrony and degree of systolic dysfunction. J Am Soc Echocardiogr 21: 214-218, 2008.

QUAN X, ZHU TG, GUO S, MA JX, WANG X, GUO JH: Ventricular synchronicity: observations comparing pulse flow and tissue Doppler assessment in a Chinese healthy adult cohort. Chin Med J (Engl) 125: 27-32, 2012.

ROULEAU F, MERHEB M, GEFFROY S, BERTHELOT J, CHALEIL D, DUPUIS JM, VICTOR J, GESLIN P: Echocardiographic assessment of the interventricular delay of activation and correlation to the QRS width in dilated cardiomyopathy. Pacing Clin Electrophysiol 24: 1500-1506, 2001.

SADEGHIAN H, AHMADI F, LOTFI-TOKALDANY M, KAZEMISAEID A, FATHOLLAHI MS, GOODARZYNEJAD H: Ventricular asynchrony of time-to-peak systolic velocity in structurally normal heart by tissue Doppler imaging. Echocardiography 27: 823-830, 2010.

SANTOS M, SHAH AM: Alterations in cardiac structure and function in hypertension. Curr Hypertens Rep 16: 428, 2014.

SCHUSTER I, HABIB G, JEGO C, THUNY F, AVIERINOS JF, DERUMEAUX G, BECK L, MEDAIL C, FRANCESCHI F, RENARD S, FERRACCI A, LEFEVRE J, LUCCIONI R, DEHARO JC, DJIANE P: Diastolic asynchrony is more frequent than systolic asynchrony in dilated cardiomyopathy and is less improved by cardiac resynchronization therapy. J Am Coll Cardiol 46: 2250-2257, 2005.

SURAWICZ B, CHILDERS R, DEAL BJ, GETTES LS, BAILEY JJ, GORGELS A, HANCOCK EW, JOSEPHSON M, KLIGFIELD P, KORS JA, MACFARLANE P, MASON JW, MIRVIS DM, OKIN P, PAHLM O, RAUTAHARJU PM, VAN HERPEN G, WAGNER GS, WELLENS H: AHA/ACCF/HRS recommendations for the standardization and interpretation of the electrocardiogram: part III: intraventricular conduction disturbances: a scientific statement from the American Heart Association Electrocardiography and Arrhythmias Committee, Council on Clinical Cardiology; the American College of Cardiology Foundation; and the Heart Rhythm Society: endorsed by the International Society for Computerized Electrocardiology. Circulation 119: e235-e240, 2009.

SYNEK V: Evaluation of the standard deviation from duplicate results. Accred Qual Assur 13: 335-337, 2008.

TROUGHTON RW, RICHARDS AM: B-type natriuretic peptides and echocardiographic measures of cardiac structure and function. JACC Cardiovasc Imaging 2: 216-225, 2009.

VINEREANU D, KHOKHAR A, FRASER AG: Reproducibility of pulsed wave tissue Doppler echocardiography. J Am Soc Echocardiogr 12: 492-499, 1999.

WANG J, KURRELMEYER KM, TORRE-AMIONE G, NAGUEH SF: Systolic and diastolic dyssynchrony in patients with diastolic heart failure and the effect of medical therapy. J Am Coll Cardiol 49: 88-96, 2007.

YU CM, LIN H, HO PC, YANG H: Assessment of left and right ventricular systolic and diastolic synchronicity in normal subjects by tissue Doppler echocardiography and the effects of age and heart rate. Echocardiography 20: 19-27, 2003.

YU CM, ZHANG Q, YIP GW, LEE PW, KUM LC, LAM YY, FUNG JW: Diastolic and systolic asynchrony in patients with diastolic heart failure: a common but ignored condition. J Am Coll Cardiol 49: 97-105, 2007.

YU CM, BAX JJ, GORCSAN J 3RD: Critical appraisal of methods to assess mechanical dyssynchrony. Curr Opin Cardiol 24: 18-28, 2009. 